\title{
Challenges in the Development of Therapeutics and Vaccines for Covid 19.
}

\section{${ }^{1}$ Nadarajah Sreeharan}

\author{
${ }^{1}$ Visiting Professor, Kings College, London \& University of Jaffna, Sri Lanka
}

The Covid 19 (COV) pandemic has spread rapidly around the globe and the associated morbidity and mortality have been staggering. (1). Feelings of despair, fear and anxiety have arisen from the considerable uncertainty a new virus has unleashed into the global society, especially in the absence of approved therapies or a vaccine. The main modality to manage and control the spread of the virus has been the stringent public measures, including physical distancing, lock downs and travel restrictions. Repositioning some existing medications with well-defined benefit-risk profiles in other indications have also been attempted but many of these have limited evidence on the benefit-risk in COV patients, obtained mainly from in-vitro studies or inadequately powered clinical studies. It is now clear that an effective vaccine will be needed to bring the global pandemic under control. However, there are significant issues and challenges in the task of finding new therapeutics and vaccines for COV. Until the availability of effective therapeutics or vaccines, it is essential for societies to learn to adapt and manage the uncertainties of the post Covid era.

\section{Process For Drug Development In A Pandemic}

The absence of approved therapies and vaccines for COV has resulted in a rapid and exponential expansion of new research endeavours. Under normal circumstances, the drug and vaccine development processes are complex, prolonged over many years and filled with challenges. The development takes place sequentially from the laboratory through preclinical testing of lead molecules and through in-vitro and in-vivo animal studies before entering clinical trials in humans. The clinical development proceeds from early phase 1 trials in healthy volunteers, followed by Phase 2 trials in small numbers of the target population to identify the "proof of concept" (preliminary evidence of benefit and risk) before embarking on large scale confirmatory Phase 3 trials in thousands of patients (for therapeutics) and the population at risk (for vaccines). The process has rigorous regulatory oversight to ensure that the benefit- risk profile of the intervention is acceptable at every stage of development and before the therapeutics or vaccines are approved for wider use. However, we are in the middle of a global pandemic and it is not "business as usual". Innovative research, development and regulatory measures are needed to expedite the process without compromising the safety of the treated population. As of early June 2020, the development pipeline across many countries included over 1000 clinical trials on therapeutics (2) and well over 100 Vaccine leads, including 8 in the clinic.(3) The Drug and Vaccine development processes are being conducted in parallel to expedite the process, but this requires care, control, and prudent decision making. Global pandemics also need global solutions and it is imperative that all stakeholders, including clinical scientists, industry, regulatory agencies, governments and non-governmental organizations, should come together to optimize the efforts. Although significant concerns remain with a somewhat disjointed nature of the search for medicines and vaccines around the world, resembling more of a cacophony than a symphony, recent efforts to create national and global collaborative partnerships are to be welcomed (4). These partnerships should ensure the use of standardized protocols and study designs and help establish global clinical trial networks to 
speed up the conduct and recruitment into studies. Enrolling trial subjects into standardized global protocols will ensure that single multi-center trials or a meta-analysis of several trials will have the necessary power to arrive at firm conclusions of the benefit-risk of the interventions under investigation. It is also essential to recruit study sites from non-High-Income countries into the international studies to ensure acceptability and relevance across the global population (5). Such an effort will also prevent individual countries embarking on inefficient, small, and underpowered studies.

Avenues are available during pandemics for the global regulatory agencies such as the US Food \& Drug Administration (FDA) and European Medicines Agency (EMA) to invoke special measures $(6,7)$ to accelerate or short circuit some of the regulatory requirements and timelines to ensure the rapid access to medicines and vaccines.

\section{Development of Therapeutics}

All medical interventions including repositioned therapies. should be considered as "experimental", until clear evidence emerges of their utility or harm in COV (8). Any Medicine (e.g. hydroxychloroquine) which has been found to have an acceptable benefit-risk in some diseases (e.g. malaria, lupus, rheumatoid arthritis) does not automatically become an acceptable medicine for a new indication (i.e. Covid 19). Recent data from clinical trials, including in post-exposure prophylaxis in high risk subjects (9) and hospitalised patients (10) have not demonstrated any clinical benefit for hydroxychloroquine. The dangers of accelerating research and publications even from reputable academic units and leading journals came to light when one of the published studies was retracted from the Lancet soon after the original publication. Data on ongoing studies assessing hydroxychloroquine as pre-exposure prophylaxis in frontline healthcare workers are still awaited.

An understanding of the clinical phases of COV (11) has enabled the targeting of appropriate therapeutic interventions to the relevant patient populations. Recent data demonstrating mortality benefits with low dose dexamethasone for hospitalised patients requiring $\mathrm{O}_{2}$ therapy or ventilatory support could be ground-breaking (12). It also offers "proof of concept" for other anti-inflammatory agents that are currently being tested, including potential modulators of the cytokine release syndrome (CRS) such as Interleukin 6 antibodies and JAK inhibitors. Although, the preliminary data from Remdesevir has been encouraging in Stage 2 of the disease demonstrating a shortening of the duration of stay in hospital (13), further data are needed to confirm any mortality benefit. In addition to repositioning of existing medicines, the increased understanding of the molecular structure of SARS-CoV 2 and the mechanisms underlying its pathogenicity in humans has allowed the research and development of new molecular entities specifically targeted against SARS-Cov-2, although the time frame for development of these novel medicines may be somewhat protracted. It is unlikely that any single therapeutic intervention will emerge as a "game-changer" but a combination of therapies with anti-viral, anti-inflammatory, and other "extra pulmonary" actions, such as the use of heparin to control thrombosis should have the greatest chance of offering clinical benefit.

Care should be taken in the current state of accelerated development of therapeutics and vaccines to ensure that some of the basic scientific and ethical principles are not compromised, as has been stated in the report of the WHO panel convened during the Ebola epidemic. (14). Indications that the primary endpoint of the Remdesevir trial was changed, although done with regulatory approval, could invalidate some of the conclusions and the integrity of the study. The benefit-risk profile of all interventions in development should be communicated transparently to the participating patients and the wider public.

The informed consent for all trials, especially in resource poor settings should be drafted carefully to meet the normal Good Clinical Practice 
requirements. Any interference in the regulatory process, however implicit, by politicians and governments should be resisted.

\section{Development Of Vaccines}

The development of vaccines for COV remains as the main, perhaps the only, means of a permanent solution to the pandemic. There have been several mixed messages as to the potential of each of these vaccines and the timings of their availability. The structure of the SARS-CoV-2 virus (Figure 1) highlights the main areas targeted for vaccine development. These include Live attenuated viruses, Protein sub-units mostly related to the Spike Protein and Nucleoside related (RNA) leads, administered either directly or through viral vectorbased technologies. News of any potential or early delivery of vaccines must be tempered by the existence of significant issues, challenges, and uncertainties in the delivery of safe and effective vaccines for $\mathrm{COV}$.

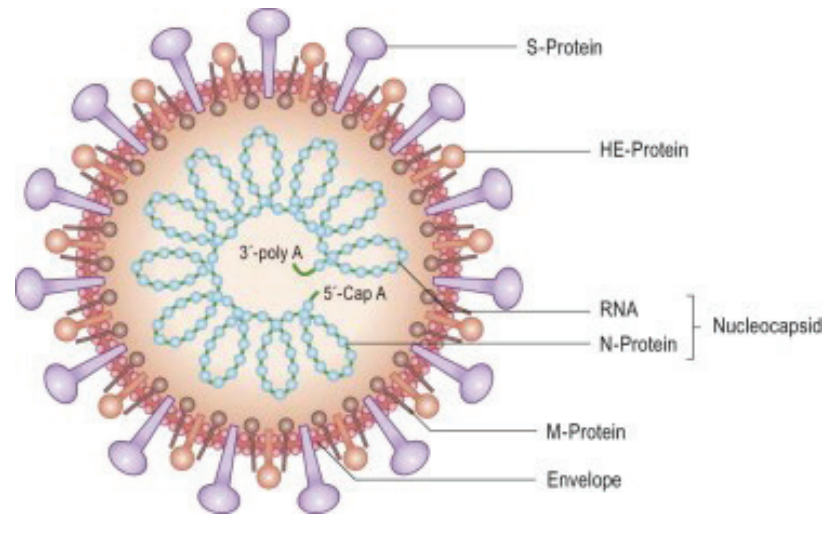

Figure 1 : Structure of SARS - CoV - 2

These are briefly highlighted below.

1. Mutation of the Virus: Although the virus has already mutated several times including at least 14 mutations of the Spike protein (15), it is difficult to predict the effects of these mutations on transmission, pathogenesis, and immune interventions. The consensus scientific opinion is that the mutations so far have not been significant enough to impact on the current vaccine leads in development. Careful tracking of the mutations and emerging strains will need to continue.
2. Risk Mitigation and Societal Acceptance of Risk: There are inherent safety risks and uncertainties in the development of a vaccine for COV. Many of the technologies, including the use of Adeno viral vectors and the immunogenic responses of the family of corona viruses remain untested. The risk of any vaccine under development will only be mitigated progressively with the continued accumulation of benefitrisk information during the development process. Until the vaccine can provide a full complement of evidence (Completed Vaccine), any vaccine should be considered as a "Developmental Vaccine". The level of risk of the vaccine that is acceptable to the regulators and indeed the public will determine the time of release of the vaccine for wider use. Generally, compared to therapeutics, a higher level of benefitrisk profile is required for vaccines which are administered to relatively healthy populations. From a regulatory standpoint, the developmental vaccines could be released with varying risk profiles either "approved" by the regulators or more likely, through the "emergency provisions" outlined earlier.

\section{Antibody Response and Protectivity:} There is currently limited information on the strength, duration, and nature of the antibody response. Although the presence of an antibody response from the sera of recovered Covid patients offers some optimism, further assessment of the antibody response is needed, including in the elderly, different ethnic groups and in those with comorbidities. Furthermore, the presence of the antibody response does not equate with protection from the virus and more work is needed to understand the roles of "neutralising antibodies" and the cell mediated response in providing longer term protection. Recent Phase 1 data in a young and healthy population (16) demonstrating 
good levels of neutralising antibodies and $\mathrm{T}$ cell responses to the spike protein is encouraging, and data from the ongoing Phase 3 trials will be needed to demonstrate the level of protection against Covid 19, especially in the high risk and elderly population. Since the clinical course of the virus has been punctuated by the occurrence of exaggerated immunological response and "cytokine storms", it is essential that the safety of any vaccine should be continuously monitored to detect the presence of the rare complication of "antibody dependent enhancement" as was the case with the vaccine for Dengue fever (17)

\section{Access and Affordability of the Vaccine.} Manufacturing of the billions of doses needed to create sufficient level of immunity in the global community to control the virus will require a coordinated effort by the industry, government, and regulators. An approximate calculation based on the $\mathrm{R}_{\mathrm{o}}$ value suggests that $2 / 3^{\text {rd }}$ of the population of the world will need to develop immunity (naturally and through a vaccine) to bring the virus under control in the absence of physical distancing measures. Reaching this target will be a long journey that will possibly need several different vaccines and a stepwise immunisation, initially commencing with the vulnerable target groups. A concerted global effort through public-private partnerships (18) will be needed to make the vaccine affordable to the population in lower income countries. Although these challenges do exist, we should also be hopeful as we live in extraordinary times and the efforts that are being made towards a Covid 19 vaccine are also unprecedented. The ability and willingness of all stakeholders to share information, including intellectual property and resources across the public and private sector gives hope that we could see some positive developments in the coming months. Some of the lessons learnt from the innovative processes and technologies that were applied on the drug and vaccine development pathways during the pandemic, would perhaps allow changes in the post-COV era to expedite the availability, access, and affordability of medicines across the world.

\section{Epilogue}

Uncertainty exists as to whether the world will ever eradicate the virus or whether it will become endemic with flareups across time and geography. Such an endemic flu-like status is likely, if either the virus mutates significantly and regularly, or if the immunity from the virus is not sustained over time. However, the pandemic could also cease to "exist" if we learn to manage both the virus and the uncertainty that leads to fear and anxiety in the minds of individuals and society. The considerable effort and investments that are being made towards the development of therapeutics and vaccines should also be extended to tackle the inequalities in our societies and in enhancing the mental well-being, especially of vulnerable populations. Potential pandemic diseases are emerging with greater frequency as climate change and the interaction of humanity with animals result in an increase in viral mutations and vector borne diseases. If the lessons from Covid 19 do not result in better preparedness for future pandemics and the creation of a society which is more just and caring, the innumerable loss of life and livelihoods would have been in vain.

\section{References.}

1. WHO Coronavirus (Covid 19) Disease Dashboards. https:// covid19. who. IVGO7tCh2BZQW0EAAYASAAEgJUGPD_ $\mathrm{BwE}$

2. Thorlund K, Dron L, Park J, Hsu G, Forrest JI, Mills EJ.A real-time dashboard of clinical trials for Covid 19. Lancet Digital Health. 2020;2(6):286-287 https://doi.org/10.1016/S2589-7500(20)30086-8 
3. WHO Draft landscape of Covid 19 candidate vaccines https://www.who.int/who-documentsdetail/draft-landscape-of-covid-19-candidatevaccines

4. Collins FS, Stoffels P, Accelerating Covid 19 Therapeutic Interventions and Vaccines (ACTIV); An Unprecedented Partnership for Unprecedented Times. JAMA. published on line 18 May 2020. doi:10.1001/jama.2020.8920

5. Dal-Re R, Carcas AJ, Sreeharan N, The need to implement non-Industry Covid 19 clinical trials in non-High-Income countries. JoGH. 2020;10(1): 010351. doi:10.7189/jogh.10.010351

6. US Food \& Drug Administration (FDA). Emergency Use Authorisation https://www.fda. gov/emergency-preparedness-and-response/ mcm-legal-regulatory-and-policy-framework/ emergency-use-authorization

7. European Medicines Agency. EMA initiatives for acceleration of development support and evaluation procedures for COVID-19 treatments and vaccines. https://www.ema.europa.eu/en/documents/other/ ema-initiatives-acceleration-development-supportevaluation-procedures-covid-19-treatmentsvaccines_en.pdf

8. Sreeharan N. Sars-Cov-19:Administering experimental medicines in a pandemic. BMJ. Rapid response on line $2^{\text {nd }}$ April 2020. https://www.bmj. com/content/368/bmj.m1091/rr-5

9. Boulware DR, Pullen MF, Bangdiwala AS et al, A Randomized Trial of Hydroxychloroquine as Postexposure Prophylaxis for Covid-19. NEJM. published on line 3rd June 2020. https://doi. org/10.1056/NEJMoa2016638

10. Horby P, Landray M on behalf of Recovery trial investigators. No clinical benefit from use of hydroxychloroquine in hospitalised patients with Covid 19. Published on line $5^{\text {th }}$ June 2020. https://www.recoverytrial.net/news/statementfrom-the-chief-investigators-of-the-randomised- evaluation-of-covid-19-therapy-recovery-trialon-hydroxychloroquine-5-june-2020-no-clinicalbenefit-from-use-of-hydroxychloroquine-inhospitalised-patients-with-covid-19.

11. Siddiqui HK, Mehra MR, COVID-19 illness in native and immunosuppressed states: A clinicaltherapeutic staging proposal. J of Heart \& Lung Transplantation. 2020;39(5): 405-407. https://doi. org/10.1016/j.healun.2020.03.001.

12. Effect of dexamethasone in hospitalized patients with COVID-19 - preliminary report. 22 Jun 2020 (preprint). https://www.medrxiv.org/ content/10.1101/2020.06.22.20137273v1.full.pdf.

13. BeigelJH,TomashekKM, DoddLEetal,Remdesivir for the Treatment of Covid-19 - Preliminary Report. NEJM. published on line 22nd May 2020. https:// doi.org/10.1056/NEJMoa2007764

14. WHO Advisory panel report on Ethical considerations for use of unregistered interventions for Ebola viral disease, 2014 https://apps.who.int/ iris/bitstream/handle/10665/130997/WHO_HIS_ KER_GHE_14.1_eng.pdf

15. Korber B, Fischer WM, Gnanakaran S et al. Spike mutation pipeline reveals the emergence of a more transmissible form of SARS-CoV-2. Preprint $5^{\text {th }}$ May 2020, BioRxiv, https://doi. org/10.1101/2020.04.29.069054

16. Foligatti PM, Ewer KJ et al on behalf of the Oxford COVOD Vaccine Trial Group. Safety and immunogenicity of the ChAdOx $1 \mathrm{nCoV}-19$ vaccine against SARS-CoV-2: a preliminary report of a phase $1 / 2$, single blind, randomized controlled trial. Lancet. Published online July 20, 2020. https://doi. org/10.1016/S0140-6736(20)31604-4

17. Halstead SB, Dengvaxia Sensitizes Seronegatives to Vaccine Enhanced Disease Regardless of Age. Vaccine. 2017; 35(47):6355-6358. https://doi. org/10.1016/j.vaccine.2017.09.089.

18. Gavi, the Vaccine Alliance. https://www.gavi.org 